\title{
The Application of Personalized Search Based on SoftMan Architecture
}

\author{
Qingzhen Wang ${ }^{1, a}$, Kang Lu ${ }^{1, b}$ \\ ${ }^{1}$ Department of Computer Science, Zhengzhou College of Science \&Technology, Zhengzhou \\ 450064, China \\ aemail: weixingji123@163.com, bemail:lukang0870@163.com
}

\begin{abstract}
Keywords: children's TV programs; intelligent search; Soft Man; structure model
\end{abstract}
\begin{abstract}
Along with the digital television popularization, television channels and programs have been becoming more substantial. Thus, this paper proposes an intelligent solution for the problem of searching TV programs when children are watching TV. After that, this paper designs search software for children to search TV programs more fast, accurate, convenient. Firstly, this paper expounds the definition of "Soft Man" and its composition. Secondly, this paper accomplishes the behavior of Soft Man for searching TV programs and builds its structure model by using VB6.0. Moreover, the design of this Soft Man can be further grafting to other Soft Man systems and application areas, and can generate the corresponding intelligent systems.
\end{abstract}

\section{Introduction}

With the application of Internet, human can intelligent gets useful information by using Internet. Nowadays, people focus on how to let computer understand instructions through simulating human thinking mode, make the relation of human-computer-Internet more coherently, and make computer and Internet serve humans better. Since the first Web software using in Steven Job's NeXT computer system in 1990[1], Web technology and its application has taken quite great development in the world and penetrated into every field of human's life. With so much Web servers and Web information resources, how to effectively acquire Web information become more and more important.

The information has been growing faster out of imagination. In the face of vast amounts of TV programs, newspapers, magazines, etc., it has become more and more difficult to accurately obtain information that you really need, and it can hardly satisfy the requirements through several Internet search tools. Among these digital TV programs, children can find a suitable program only with their parent's help, and parent also need to select suitable program from numerous TV programs. In the face of this problem, the best way is to search TV programs through Internet. However, for children, due to the restrictions of their ability of read and operation, it's difficult to find a suitable program by using search tools for them. So we try to design softMan[2] for children's television-programming search according to children or their parent's will. Therefore, we can not only help children to search specified TV programs and return the playing TV programs and its time, but also can be willing to help children to search their favorite TV program according to the children's fancies. Besides, parents can set children's favorite TV program, add or remove TV programs according to the children's fancies. In the face of these programs, this paper designs a Soft Man for searching children's favorite TV program, proposes the definition of SoftMan, the behavior realization and structure model of searching Soft Man.

\section{SoftMan}

In 2002, Prof. Xu XuYan and Zeng GuangPing had proposed the concept of "SoftMan”. In the research of thinking science, intelligence science, artificial intelligence; they gave the definition of "software man" and its state description model. In this paper, we will use this model to design children's television program search software.

1)The definition of SoftMan 
From the view of generalized artificial life, "SoftMan" has proposed to extend the life of people. This SoftMan lives in software environment, moves in network world and it is a kind of "virtual robot", which has something like human (thinking, awareness, behavior characteristics and information handling, obtain capacity). This Soft Man has some personification properties, functions, behaviors and structures. From the view of system, for living in the network, environmental factors should also be one part of this Soft Man system. The following figure 1 is the structure of this Soft Man System.



Figure 1 the composition of Soft Man

Figure 1 is a concrete manifestation of SoftMan. Among them, A is the personification properties, which includes Aauto,Aacti, Asens, Areac, Amobi and Asoci; F is the personification functions, which includes Fl, Fo and Fw; D is the personification behaviors, which includes Dadap, Devol, Dgene and Dacti; S is the personification structure, which includes Sb, Sf and Sa; E is the net environment, and so on.

2)The behavior realization of searching Soft Man

As a living body, SoftMan shows different behaviors. The behavior of the start, continuation, and stop is the track of SoftMan in the network. This Soft Man for searching children's favorite TV program has realized by parent management form and starting form through internet, as shown in figure 2 .

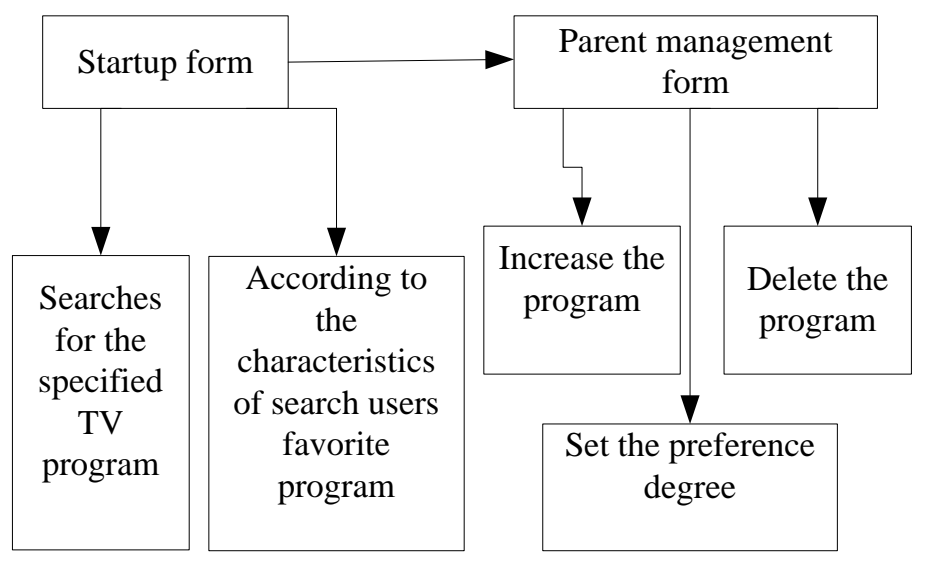

Figure 2 the functional diagram of searching SoftMan

This SoftMan starts from sub main (). For the convenience of access database, it defines a global variable of access database.

The main form does the following things: By using the function of automatic search of this searching SoftMan, it sends the search results via wireless transmission equipment like WIFI or blue tooth to a receiver for choosing TV programs. So this main form must have a concise and clear form, which enables children more easily to operate. Considering that children don't know what they love, and desire to be paid more attention, this SoftMan adds a "guess you like" function. Therefore, it can not only satisfy children's viewing requirements, but also meet the psychological needs of children's desire to be paid more attention (although this "people” is just a Soft Man).

The parent management form does the following things: add programs, alter user's feature library. Thus, it can not only be convenient for parents to manage and control children's behavior 
when they watch TV program, but also be convenient for parents to use the list box controls. Through this indirect management and supervision on watching children's TV program, it can effectively avoid direct conflict and cultivate the understanding between parents and children, thus accomplishing the purpose of human communication and interaction between parents and children.

The above introduction reflects more comprehensive personification intelligent features and behaviors about this SoftMan, and this SoftMan has the ability of environment recognition, autonomous decision-making and free will.

\section{The structure model of searching SoftMan}

The Soft Ware is an intelligent agent, it has the ability to analyze and solve problems. Therefore, the most important thing of design SoftMan is to design its information processing system and execution system. SoftMan has the ability to self-learning and self-evolution. When it is in a strange environment, or treat the strange event, Soft Man can use the experience to solve problem. If it cannot solve problem or the effect is weak, it will try to record the best way by trying used learning and associative memory method. This SoftMan searches the children's TV programs via computer, sends the results to children or parents, and switches the TV channel to watch TV programs. The whole design of Soft Man includes four parts: start, index, match and report, its interaction relationship with the Internet is shown in figure 3. The design of each part of this SoftMan uses the generalized operator model method[3]. The whole process is as follows: firstly, it collects data from the environment, filter these sensory data as filter data. Then, it makes a decision by using these methods such as cognition, learning, thinking, associative memory based on filter data. Finally, it returns behavior results by executing to the executive components according to the decision information.

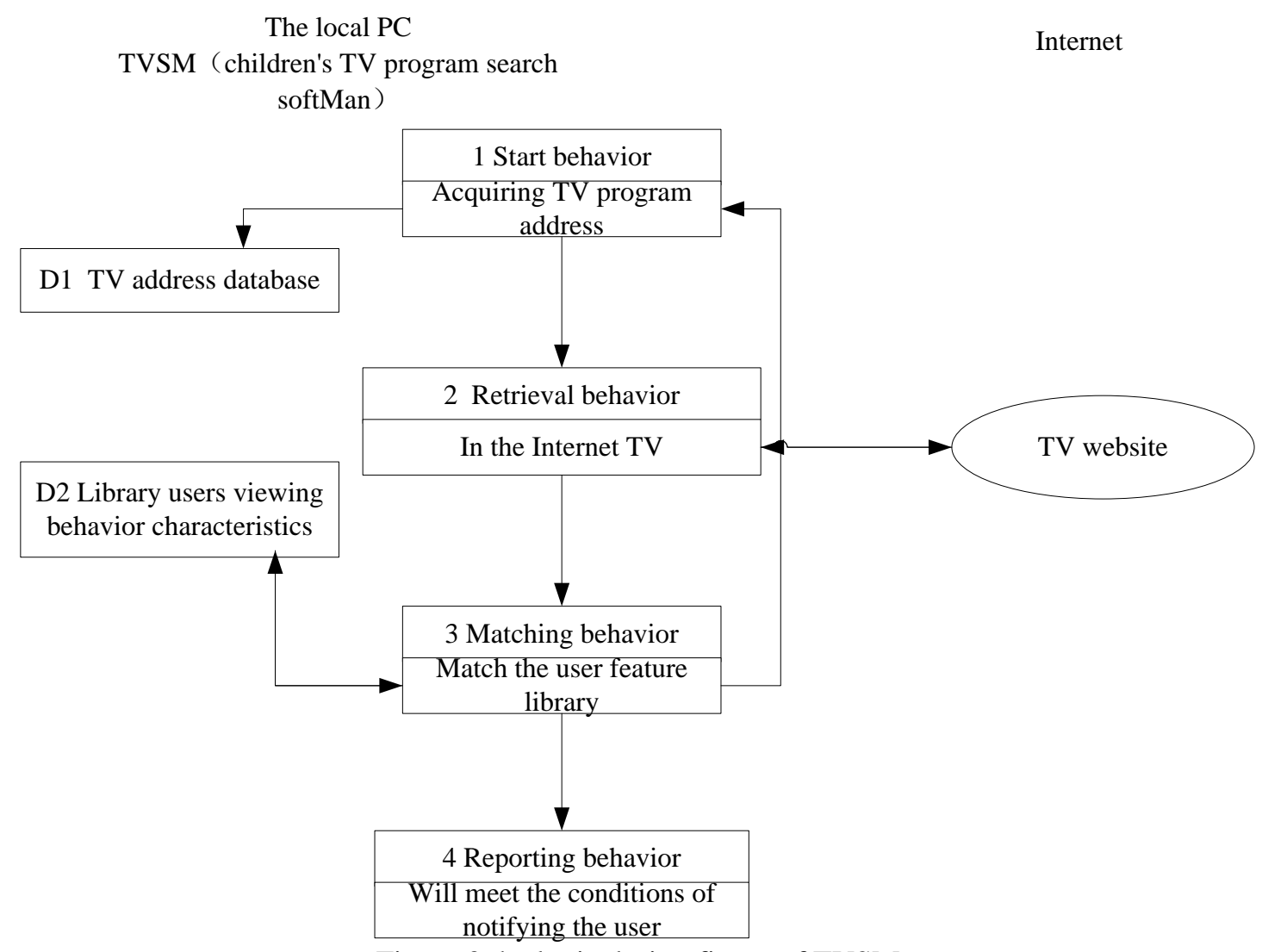

Figure 3 the logic design figure of TVSM

As SoftMan's perception system, the operation of start can make Soft Man filter the useless, excess demand for the current behavior according to the current task needs. The current task requirements can be the tasks that come from environmental change, the special task or other Soft Man's message tasks, etc. When PC is running, once SoftMan receives the search signal, the project will start sub main () and load the main form. The local television program database will be opened 
while loading the main form. After that, it will sort TV forecast program in the form of record, connect various television programs on the web, extract the text information of the whole pages as Block [4], and deposit into the corresponding string variable.

Nowadays, the set-top box can conveniently connect PC to the Internet by providing many interfaces to PC. Therefore, it's a best way to search programs via SoftMan through Internet. As an information processing component of SoftMan, the task of searching is to process those perceived data. It is the brain of SoftMan, which is mainly responsible for cognition, learning, thinking, associative memory and decision-making and other functions. Firstly, it processes those perceived data, builds cognitive model, and thinks about the problem by using the associative memory method. Then, it retrieves the corresponding data from the model library. Finally, it makes a decision.

This design uses test box to store those being searched TV programs, contrasts being searched text to the web text which extracts from variables, and queries content which stores in variables. Next, it makes use of VB6.0's function of InStrRev (), Mid (), analyzes the web page source, and computes the location of the program. Then, it obtains playing time according to its location. Because of obtaining only TV program and playing time, the amount of information is not large and there are not so much pages to query, this design extracts and analyzes the source code of the page by using WebBrowser1.Document.documentElement.outerHTML[5], uses the character of the source code to set query expression. Besides, this design extracts the text of the page by using WebBrowser1, Document Element, and Outer Text. If there are so many pages to query, we can use regular query.

With the help of a search tool to retrieve Web information, it usually returns a lot of related information. In general, common search tools only use the keyword of user's query as the only input, but the keyword itself cannot fully reflect the intention of user's query. As the operating unit of Soft Man, the match and report of SoftMan can run independently, perceive the request of external environment. It can be realized personalized search[6] through improve search quality based on user's feedback, filter and sort the search results by using the user's interest model, and capture user's potential query intentions via user's extension, etc. The user's viewing behavior characteristic library is made up of the program's love degree being set by parent and the played record of one program. By comparing the page's query results with the query results from the user characteristics list, it will automatically select user's favorite programs. If there are so many query tasks, the query that system often use can be saved as the stored procedure. If there are so much data to be returned, we can use vernier technique [7]. It has influence on the environment through the actions according to the processed results of information processing system. With the matched result, it returns the played programs and time of user's favorite program. It will return the search results to the client by using WIFI, bluetooth, or other wireless equipment. The receiving end is installed in PC and children or parents have a sending end. Children or parents can choose whenever turn on the TV and see any program they want according to the sending search results.

\section{Conclusion}

The application of this SoftMan can help children to search TV programs. With the emergence of SoftMan, we can complete web search through computer by using searching SoftMan to, select goods by using shopping SoftMan, chatting with Soft Man by using some SoftMan which can understand human language, etc. These SoftMan can move freely via Internet, automatically process some special tasks by using the technology of information push and pull[8], and act as some roles, such as Online "police”, Online "garbage collectors", Online "information server”, etc. The model of Soft Man system can graft in the field of application, and generate corresponding intelligent system. These Virtual assistant [9] are useful to help people complete daily online task and save our time. SoftMan is a new research field with strong vitality, which is born at the request of more intelligent to obtain information. Besides, this searching SoftMan can add other functions in order to realize the monitoring function for children. 


\section{Acknowledgement}

( The project of National Natural Science Fund: 61272392, Science and Technology Department of Henan province science and technology projects: 142102210511, Science and Technology Department of Henan basic and frontier technology projects: 112300410110)

\section{Reference}

[1] Yang Guangjun,Wu Yuhong. Friend or foe[M].In 2007 June.

[2] Zeng Guangping,Tu Yanxu,Wang Hongbo. "SoftMan” of Research and Application[M].Science Press.In 2007.

[3] Felipe N.Martins,Wanderley C.Celeste,Ricardo C,et al.An adaptive dynamic controller for autonomous mobile robot rrajectory tracking[J].Control Engineering Practice.2008.16: 1354-1363

[4] Song Jie,Wang Daling. Web file collecting and storing page based on Block[J]. Journal of software. 2008.17(4):277-281.

[5] Lui Binbin,Sun Xuimei.Visual Basic Programming book（The ten Annual Edition ）. People's Posts and Telecommunications Press. In 2011 January

[6] Song Yi. Research on search TV program personalization[D]. Shanghai Jiao Tong University Master Thesis Database.In 2008 January.

[7] Li Junmin,Wang Hao.SQL Server Examples of development Daquan. Tsinghua University press. In 2010 April.

[8] Tu Xuyan. Intelligent information "push and pull" technique[N]. Computer world.2000.14:787-791

[9] Riccardo S Architectures for distributed and hierarchical Model Predictive Control-A review 2009(05) doi:10.1016/j.jprocont.2009.02.003. 